\title{
Spectrokinetic characterization of photoactive yellow protein films for integrated optical applications
}

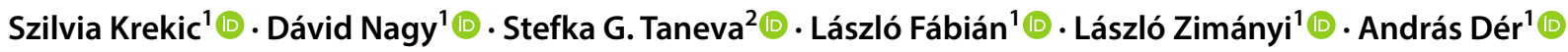

Received: 5 November 2018 / Revised: 19 February 2019 / Accepted: 4 March 2019 / Published online: 23 March 2019

(c) The Author(s) 2019

\begin{abstract}
In this paper, the photocycle of the dried photoactive yellow protein film has been investigated in different humidity environments, in order to characterize its nonlinear optical properties for possible integrated optical applications. The light-induced spectral changes of the protein films were monitored by an optical multichannel analyser set-up, while the accompanying refractive index changes were measured with the optical waveguide lightmode spectroscopy method. To determine the number and kinetics of spectral intermediates in the photocycle, the absorption kinetic data were analysed by singular value decomposition and multiexponential fitting methods, whose results were used in a subsequent step of fitting a photocycle model to the data. The absorption signals of the films were found to be in strong correlation with the measured light-induced refractive index changes, whose size and kinetics imply that photoactive yellow protein may be a good alternative for utilization as an active nonlinear optical material in future integrated optical applications.
\end{abstract}

Keywords Photoactive yellow protein · Integrated optics $\cdot$ Biophotonics $\cdot$ Kinetic absorption spectroscopy $\cdot$ Optical waveguide lightmode spectroscopy

\section{Introduction}

In the past few decades, there has been an ever increasing demand for faster information transmission and data processing. Achieving greater speed in state-of-the-art electronic devices requires further miniaturization of integrated electronic circuits and integrating more and more components on a small silicone chip. However, scaling down the structure causes several problems (heat damage, crosstalk, quantum effects, etc.), limiting the size of the components. The empirical law proposed by Moore (1965), stating that the number of electronic components integrated on a silicon wafer of unit area is doubling in every 18 months, has been

Special Issue: Regional Biophysics Conference 2018.

Szilvia Krekic

krekic.szilvia@brc.mta.hu

1 Institute of Biophysics, Biological Research Centre, Hungarian Academy of Sciences, Temesvári krt. 62, P.O. Box 521, Szeged 6701, Hungary

2 Institute of Biophysics and Biomedical Engineering, Bulgarian Academy of Sciences, Acad. G. Bonchev Str., bl. 21, Sofia 1113, Bulgaria valid until recent times, but cannot be sustained for much longer (see Waldrop 2016 as a quick overview).

One of the several alternative solutions investigated is integrated optics (IO), which comprises similar elements to integrated electronics; however, in IO the information transmission and processing is done solely by optical means. Analogously to their electronic counterparts, IO circuits also comprise passive and active elements, corresponding to the wires, resistors and capacitors, on the one hand, and the transistors, on the other. The theory of integrated optics and the technology for manufacturing passive IO elements (i.e. miniature waveguides) are well established, and the main challenge is to find or develop materials with suitable nonlinear optical (NLO) properties that can function as active elements in IO circuits.

In recent works, cost-effective organic materials have been proposed to be tested as active IO components, as alternatives to the currently used semiconductor materials (Haque and Nelson 2010). The design and application of organic, pi-conjugated molecular materials generated the most interest (Hales et al. 2010; Hu et al. 2008; Service 1995), but their naturally occurring, stable counterparts, such as chromoproteins, have also been successfully tested (as a recent review, see Fábián et al. (2015)). One of the most 
researched biological materials in the field is the light-sensitive protein bacteriorhodopsin (bR) (Oesterhelt and Stoeckenius 1971, 1973), due to its relatively large light-induced refractive index change (Ormos et al. 2002) and mechanical stability (Hristova et al. 1984). In previous works of our group, the usability of bR in IO circuits has been investigated extensively, demonstrating the ability of fast optical switching and light modulation (Dér et al. 2007; Fábián et al. 2010, 2011; Mathesz et al. 2013). In our current work, similarly to $\mathrm{bR}$, the expedience of the photoactive yellow protein (PYP) in IO circuits has been investigated.

PYP is a small, water-soluble cytoplasmic protein that was first extracted from the purple halophilic bacterium Halorhodospira halophila (Meyer 1985; Meyer et al. 1987). The chromophore of the PYP is $p$-coumaric acid, which is responsible for the colour of the protein that, when excited by light, undergoes a reversible trans-cis isomerization around its thiol-ester linked bond attached to cysteine-69 (Baca et al. 1994; Hoff et al. 1994a; Van Beeumen et al. 1993). The photoexcitation is also linked to the negative phototaxis detected in bacteria where the PYP is present as photoreceptor (Sprenger et al. 1993). Upon photoisomerization PYP undergoes a cyclic reaction series between quasi-stable intermediate states, accompanying transient protonation and conformational changes before returning to its original state ("pG"). The intermediate states of the photocycle may also differ spectrally. The pG initial or dark state has an absorption maximum at $446 \mathrm{~nm}$, while during the photocycle, two spectrally distinct states are present: the redshifted $\mathrm{pR}$, followed by the blue-shifted $\mathrm{pB}$. Since the first study of the photocycle (Meyer et al. 1987, 1989), the kinetic and spectral properties of the intermediates have been investigated with numerous methods under various environmental conditions, resulting in several distinctive photocycle schemes and rate coefficients, including spectrally "silent" transitions, too (Borucki et al. 2006; Cusanovich and Meyer 2003; Groot et al. 2003; Hellingwerf et al. 2003; Hoff et al. 1994b; Ihee et al. 2005; Imamoto and Kataoka 2007; Joshi et al. 2005; Kim et al. 2012; Pande et al. 2016; Ujj et al. 1998; van der Horst et al. 2005; Yang et al. 2017). A recent work suggests that the protein's photodynamics also depend on the wavelength of the excitation light (Mix et al. 2018). Taking into account all internal and external factors, the photocycle can occur multiple ways.

When considering PYP for IO applications, the most important features are the fast spectral transitions of the protein at the beginning of its photocycle and the accompanying refractive index changes. Contrary to bR that is available in large membrane fragments, or in a detergent-solubilized form, PYP is water-soluble per se, which offers unique opportunities for its combination with passive IO elements. These properties make PYP a good candidate for using it as an active element of IO structures. For such applications, dry samples are preferred, because of their higher mechanical stability and larger photoinduced refractive index changes.

In our current work, we investigated the photocycle of dry PYP films and determined the accompanying light-induced refractive index changes at various relative humidities in order to evaluate their potential IO adaptability. For the photocycle measurements, we used an optical multichannel analyser (OMA) set-up, while the refractive index change measurements were performed with the optical waveguide lightmode spectroscopy (OWLS) method. Our results show that PYP films of controlled relative humidity are good candidates for utilization as active elements in IO devices.

\section{Materials and methods}

\section{Sample preparation}

PYP was a kind gift of Dr. John Fitch. The lyophilized protein was dissolved in $10 \mathrm{mM}$ Tris buffer, $\mathrm{pH} 8.2$, up to a protein concentration of $50 \mu \mathrm{M}$. In both experiments, $15 \mu \mathrm{l}$ protein solution was homogeneously dried on a glass slab forming an approximately $5 \mathrm{~mm}$ diameter patch. For kinetic absorption spectroscopy, the glass slab with the dried protein film was placed into a plastic cuvette. Saturated salt solutions were introduced in the bottom of the cuvette, without contact with the PYP film, to maintain the desired humidity. For the low-relative humidity (20\%) experiments, we used potassium acetate, while high-relative humidity (85\%) was achieved by using potassium chloride.

For the OWLS experiments, the protein solution was dried on the top of a slab optical waveguide directly over the grating coupler. The waveguide and the rotational table of the experimental set-up were placed inside a glass case, where the environment's humidity was also controlled with the same salt solutions as used in the absorption kinetics experiments. An additional experiment was performed in an environment where the relative humidity was kept at $75 \%$ using sodium chloride.

Samples were prepared 1 day before the experiments to attain the desired humidity and hydration state of the protein.

\section{Kinetic absorption spectroscopy}

Excitation of the protein film was done by a Surelite I NdYAG laser with an OPO extension (Continuum, USA). 5-ns pulses, with $5 \mathrm{~mJ} / \mathrm{cm}^{2}$ at a wavelength of $450 \mathrm{~nm}$, were used, while the white measuring light was provided by a $35-\mathrm{W}$ high-pressure Xenon lamp (Hamamatsu, Japan), which was chopped by a Uniblitz digital shutter to avoid excess exposure of the sample. The shutter was open for $20 \mathrm{~ms}$, and a new recording was done every $6 \mathrm{~s}$ to ensure that the protein returned to its dark state between consecutive actinic 
pulses. The excitation pulse and the measuring light crossed the sample perpendicular to each other. Absorption difference spectra were detected with an Andor iStar gated ICCD detector (Andor Technology, UK) which was attached to a HR-320 spectrograph (ISA Jobin-Yvon, France). The length of the gate pulse of the detector was adjusted depending on its time delay after the laser pulse, and 10-30 spectra were averaged at each delay.

\section{Data analysis}

The data analysis of the OMA experiments was based on the analysis and results in our previous article (Khoroshyy et al. 2013).

The measured time-resolved difference spectra were collected in a $\boldsymbol{D}_{\boldsymbol{m \times n}}$ matrix, where $m$ is the number of data points on the wavelength scale and $n$ is the number of different time delays after the pulsed excitation. Using the Beer-Lambert law, the matrix consisting of the difference spectra can be written as the product of the intermediate difference spectra and the transpose of their time-dependent concentrations.

Singular value decomposition (SVD) was performed on the data matrix to determine the number of spectrally distinguishable intermediates, which is equal to the rank of the matrix, and to reconstruct the data matrix with reduced noise from the significant spectral and kinetic eigenvectors. Assuming first-order transitions between the intermediates, their concentrations are composed of the linear combinations of time-dependent exponentials. As a consequence, the temporal SVD eigenvectors are also linear combinations of the same exponential functions. Multiexponential least squares fit of the temporal eigenvector matrix yielded amplitudes and phenomenological rate coefficients. The data matrix itself was then reconstructed as the linear combination of the obtained time-dependent exponentials.

A spectrotemporal least squares model fit was performed to the matrix $D^{e}$, i.e. the spectra that were reconstructed from the exponential fit of the significant SVD kinetic vectors. The fitting parameters consisted of the spectra of the photocycle intermediates and the rate coefficients of our photocycle scheme obtained by Khoroshyy et al. (2013) for the PYP in solution, and the protein fraction entering into the photocycle. The spectral fitting parameters were permitted to moderately vary relative to the input spectra, with the constraint of no negative absorption, to allow spectral differences between the photocycle intermediates observed in aqueous solution or in the dry, rehydrated sample. As the result of the fit, we could determine the spectra of the different intermediates in the photocycle and the molecular rate coefficients represented in our scheme and also calculate the concentration matrix, i.e. the kinetics of the intermediates. These data were then used in the interpretation of the refractive index changes during the photocycle measured by optical waveguide lightmode spectroscopy.

\section{Optical waveguide lightmode spectroscopy}

To determine the relative refractive index change of the PYP intermediates at different time delays after excitation, we used the optical waveguide lightmode spectroscopy (OWLS) method, which uses the principle that only such discrete modes can propagate in an optical waveguide that meet the criteria of the (1) and (2) mode equations.

$$
\begin{gathered}
\frac{2 \pi}{\lambda_{0}} d_{\mathrm{F}} \sqrt{n_{\mathrm{F}}^{2}-N_{\mathrm{TE}}^{2}}=\arctan \sqrt{\frac{N_{\mathrm{TE}}^{2}-n_{\mathrm{S}}^{2}}{n_{\mathrm{F}}^{2}-N_{\mathrm{TE}}^{2}}+\arctan \sqrt{\frac{N_{\mathrm{TE}}^{2}-n_{\mathrm{A}}^{2}}{n_{\mathrm{F}}^{2}-N_{\mathrm{TE}}^{2}}}+m \pi} \\
\frac{2 \pi}{\lambda_{0}} d_{\mathrm{F}} \sqrt{n_{\mathrm{F}}^{2}-N_{\mathrm{TM}}^{2}}=\arctan \left[\frac{n_{\mathrm{F}}^{2}}{n_{\mathrm{S}}^{2}} \sqrt{\left.\frac{N_{\mathrm{TM}}^{2}-n_{\mathrm{S}}^{2}}{n_{\mathrm{F}}^{2}-N_{\mathrm{TM}}^{2}}\right]}\right. \\
+\arctan \left[\frac{n_{\mathrm{F}}^{2}}{n_{\mathrm{A}}^{2}} \sqrt{\frac{N_{\mathrm{TM}}^{2}-n_{\mathrm{A}}^{2}}{n_{\mathrm{F}}^{2}-N_{\mathrm{TM}}^{2}}}\right]+m \pi
\end{gathered}
$$

In the above equations, $\lambda_{0}$ is the vacuum wavelength of the light propagating inside the guiding layer with a refractive index of $n_{\mathrm{F}}$ and thickness of $d_{\mathrm{F}}$, while the substrate's refractive index is $n_{\mathrm{S}}$. The $m$ is the mode order which was zero in all of our experiments. The propagating modes are also dependent on the refractive index of the adlayer $\left(n_{\mathrm{A}}\right)$, which is the material that is above the guiding layer. In our experiments, the PYP film was the adlayer. The propagation properties of the guided modes can be represented by the effective refractive indices, which are $N_{\mathrm{TE}}$ for the transversal electric (s-polarized) and $N_{\mathrm{TM}}$ for the transversal magnetic (p-polarized) modes.

Our experimental set-up was arranged in a way that the change of the effective refractive index of the guided mode could be easily determined by measuring the incident angle of the light coupled into the waveguide. The arrangement consisted of a slab optical waveguide fixed on a high-resolution $\left(\sim 10^{-3 \circ}\right)$ rotational table. As a probe we used a $\mathrm{HeNe}$ laser $(632.8 \mathrm{~nm})$, which was incident on the integrated grating coupler. This wavelength stands outside of the absorption bands of all the intermediate states, so no absorption change interferes with the measurement of the refractive index change. The incoupled light intensity was measured by two photodiodes that were attached to the two ends of the waveguide.

The change in the adlayer's refractive index alters the coupling conditions in Eqs. (1) and (2). Based on the measured angle shifts of the incoupling peak, the refractive index change could be determined up to a precision of $10^{-5}$. 
We measured the light-induced refractive index change of the dried PYP film with both continuous and pulse excitations. For the $\mathrm{CW}$ excitation, a diode laser of $410 \mathrm{~nm}$ was used. The pulse excitation was done by a $\mathrm{XeCl}$ excimer laser-pumped Coumarin 450 dye laser (wavelengths of 308 and $451 \mathrm{~nm}$, respectively).

\section{Results and discussion}

\section{Kinetic absorption spectroscopy}

The absorption difference spectra were measured in the case of both high humidity and low humidity, but extensive data were only measured when the protein was in a state of high hydration. Our analysis is demonstrated on the high-humidity environment case.

Difference spectra were recorded in the range of $250 \mathrm{~ns}$ to $1 \mathrm{~s}$ in 34 logarithmically equidistant time delays after excitation. SVD yielded a rank of 2 , based on the singular values and the autocorrelation of the spectral and kinetic eigenvectors. The first 2 eigenvectors accounted for $96.72 \%$ of the variance of the data matrix. Similar experiments on PYP in solution, in the presence or absence of various salts, typically yielded a rank of 3 (Khoroshyy et al. 2013). As it became clear from the analysis, the difference is not due to a reduced number of intermediates, but to the lack of significant spectral difference between certain intermediates in the hydrated PYP film. Global multiexponential fit was done on the two weighted kinetic eigenvectors. For an adequate fit, 5 exponentials were required, similarly to the case of PYP aqueous solutions (Khoroshyy et al. 2013). The significant $\boldsymbol{U}$ spectral eigenvectors and the corresponding $\boldsymbol{V}$ vectors are shown in Fig. 1, together with the multiexponential fit of the latter.

The five exponential amplitude spectra (B-spectra) corresponding to the multiexponential fit (Fig. 2) are by and large similar to those obtained for PYP in solution (cf. Fig. 3A in Khoroshyy et al. 2013). This indicates that not only the number of distinguishable photocycle intermediates (5) but also their spectral characteristics and the photocycle scheme are similar. The corresponding phenomenological rate coefficients are, however, different: $4.8 \cdot 10^{4}, 7.0 \cdot 10^{2}$, $1.44 \cdot 10^{2}, 57$ and $1.7 \mathrm{~s}^{-1}$, as compared to $3.43 \cdot 10^{5}, 4.0 \cdot 10^{3}$, $6.78 \cdot 10^{2}, 2.28$ and $0.18 \mathrm{~s}^{-1}$ in $0.66 \mathrm{M} \mathrm{NaCl}, \mathrm{pH} 8.2,22^{\circ} \mathrm{C}$. Since the phenomenological rate coefficients are functions of the molecular rate coefficients, the latter are also expected to be different in the dried, hydrated PYP sample compared to the aqueous sample.

Due to the overall similarity of the two data sets, we fitted the photocycle scheme published in Khoroshyy et al. (2013) (Fig. 3) to the present data. As in our earlier publication, $\mathrm{pR}_{\mathrm{O}}$
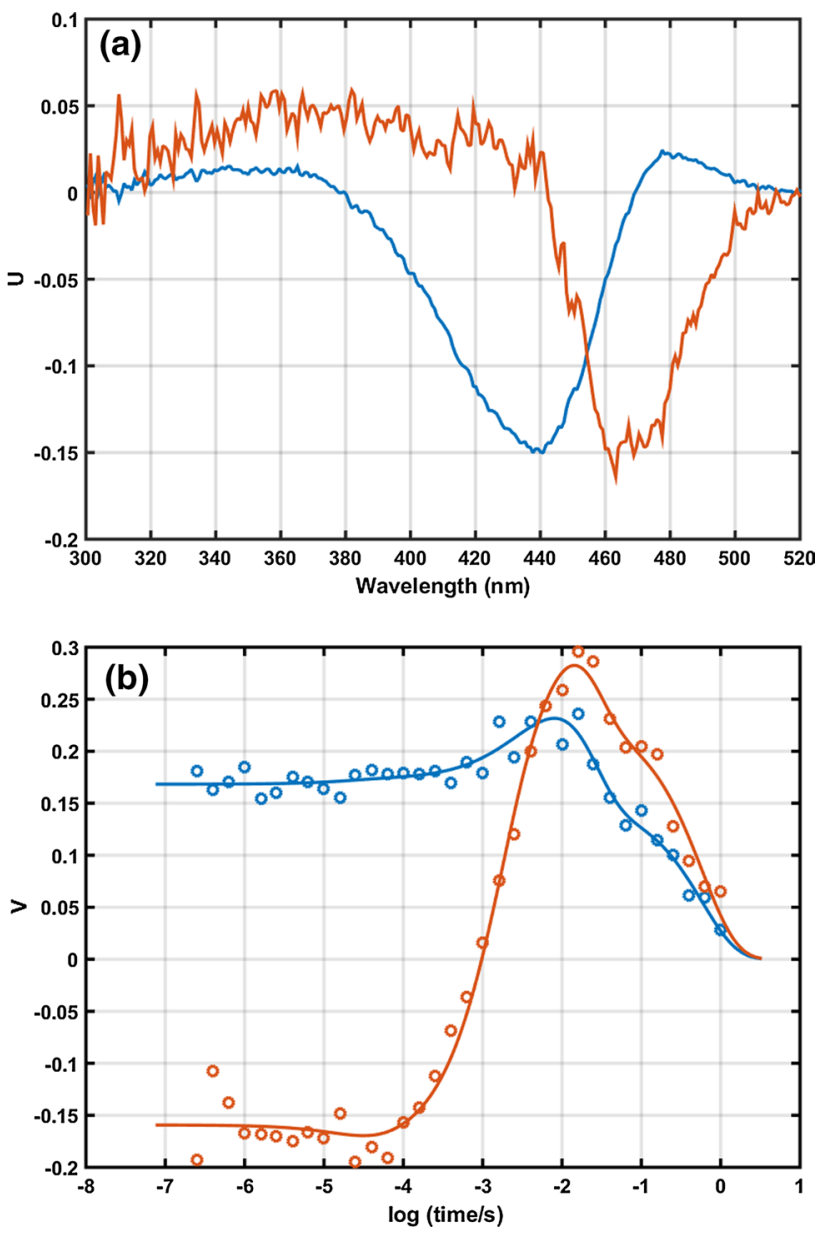

Fig. 1 The two significant spectral eigenvectors a $U_{1}$ : blue, $U_{2}$ : red; and the two significant kinetic eigenvectors $\mathbf{b} V_{1}$ : blue symbols, $V_{2}$ : red symbols from the SVD analysis. Lines in $\mathbf{b}$ show the result of the multiexponential fit to the kinetic eigenvectors

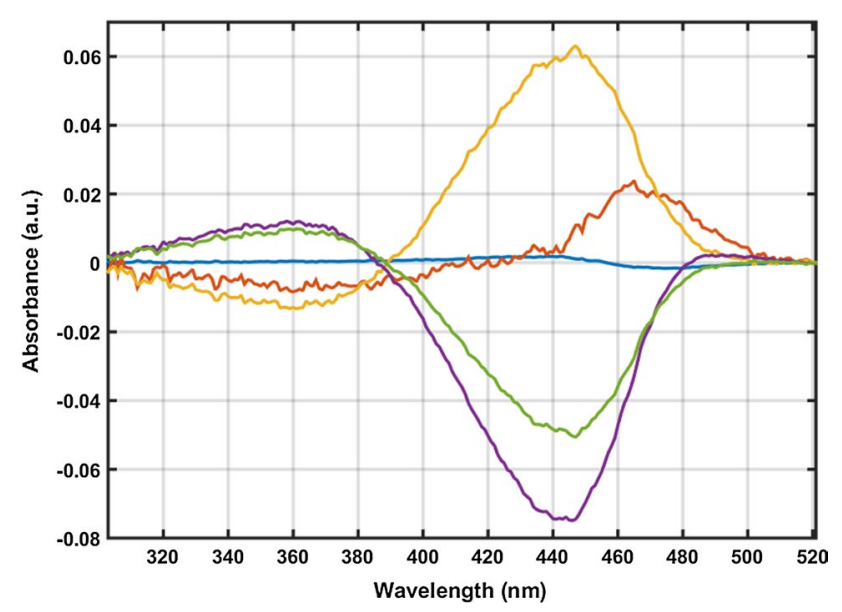

Fig. 2 Consecutive exponential amplitude spectra (B-spectra) of the multiexponential fit: blue, first component corresponding to the first (fastest) time-dependent exponential (rate coefficient $k_{1}$ ), red $\left(k_{2}\right)$, yellow $\left(k_{3}\right)$, purple $\left(k_{4}\right)$, green $\left(k_{5}\right)$ 


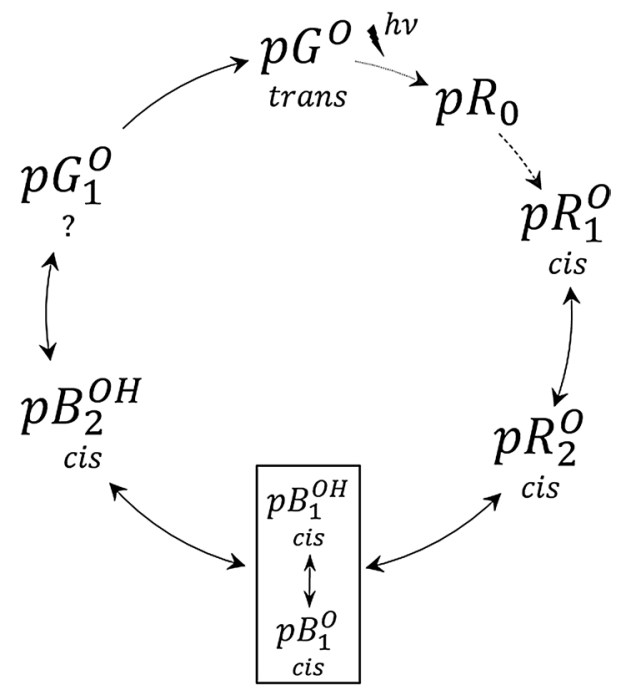

Fig. 3 The photocycle scheme. pR and pB intermediates are red- and blue-shifted, respectively, relative to the initial pG states. Trans and cis refer to the isomerization state of the p-coumaric acid chromophore of $\mathrm{PYP}$, and $\mathrm{O}$ and $\mathrm{OH}$ refer to the deprotonated and protonated states of the chromophore, respectively

was not resolved in this experiment and not included in the photocycle fit.

The fit yielded the spectra and the kinetics of the photocycle intermediates (Fig. 4a, b) and the molecular rate coefficients of the forward and reverse transitions in the scheme. The obtained intermediate spectra are similar to those published earlier (Khoroshyy et al. 2013, Fig. 4b) with two very similar $\mathrm{pR}$ spectra, with $\mathrm{pB}_{1}$ appearing as a mixture, indicating a rapid equilibrium between the protonated and deprotonated forms of the chromophore, with a distinct $\mathrm{pB}_{2}$ form and a spectrally silent $\mathrm{pG}_{1}$ as the final intermediate before the recovery of the dark state, $\mathrm{pG}$. A detailed description of the photocycle intermediates is presented in Khoroshyy et al. (2013). Briefly, the gradual conformational change (opening) of the protein starts already during the $\mathrm{pR}_{1}$ to $\mathrm{pR}_{2}$ transition, with a very minor spectral change not clearly resolved in this study. The blue shift of the spectrum is known to be due primarily to the protonation of the chromophore. It has also been shown (Khoroshyy et al. 2013) that the main conformational change appears during the $\mathrm{pR}_{2}$ to $\mathrm{pB}_{1}$ transition. The existence of the $\mathrm{pG}_{1}$ intermediate with a spectrum similar to that of $\mathrm{pG}$, the initial form, is supported by the biphasic recovery of the initial form (decay of $\mathrm{pB}$ ) without a clear spectral signature. The $\mathrm{pG}_{1}$ to $\mathrm{pG}$ transition is expected to complete the recovery of the initial conformation of the protein without affecting the immediate surroundings of the chromophore. The main difference as compared to the aqueous sample is that $\mathrm{pB}_{2}$ is not blue-shifted relative to $\mathrm{pB}_{1}$. The approximately $15 \mathrm{~nm}$ blue shift in solution has been explained by the completion of the major conformational
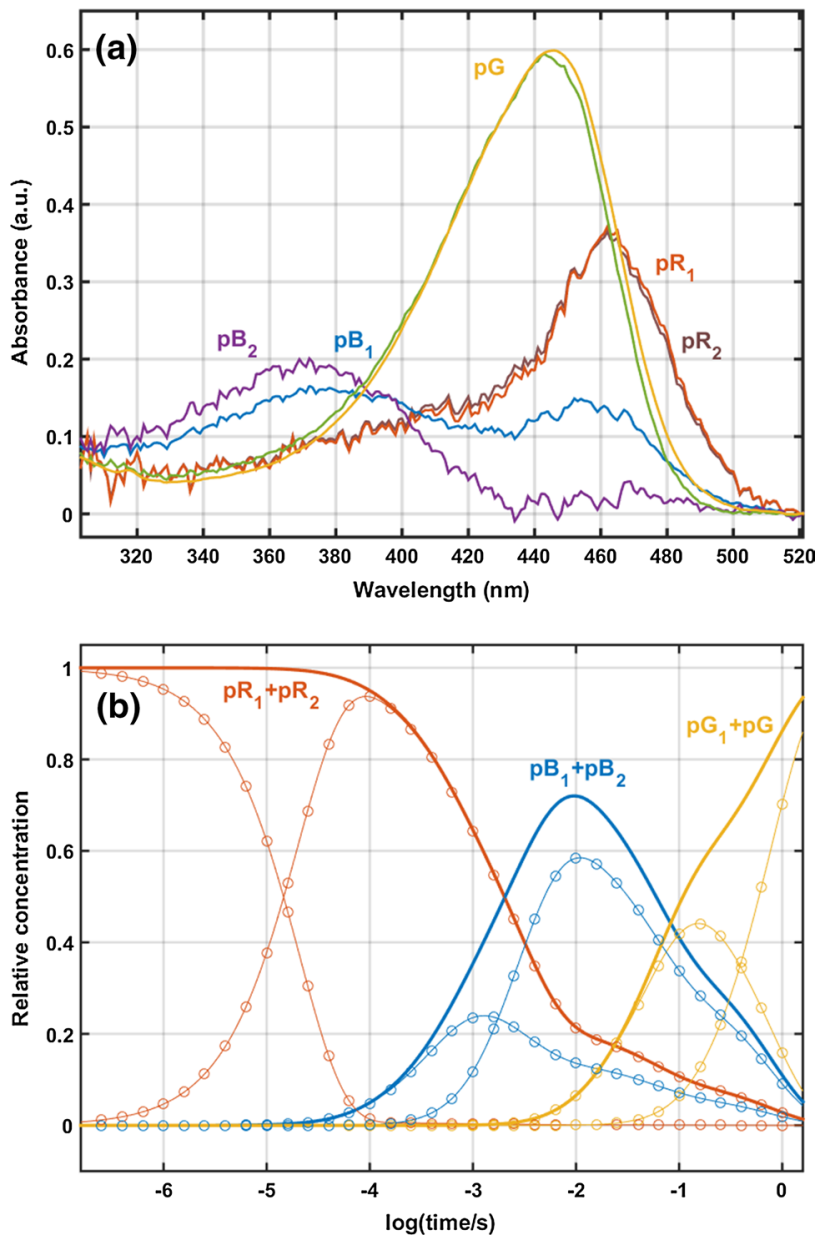

Fig. 4 The intermediate spectra obtained from the global spectrotemporal model fit (a) and the time-dependent relative concentrations of the intermediates (b). In b, thin lines show the kinetics of the consecutive individual intermediates and thick lines the total time evolution of the spectrally similar intermediates

change in the photocycle, resulting in stronger hydrogen bonding or hydration of the chromophore (Hendriks et al. 2003). This transition has not been observed in PYP crystals (Yeremenko et al. 2006), and it appears to be absent in the dried, partially hydrated PYP film, too. The absence of this conformational change in the present experiment is the probable reason for the faster recovery of the dark state, already reflected in the one order of magnitude faster fifth phenomenological exponential component, $1.7 \mathrm{~s}^{-1}$ vs. $0.18 \mathrm{~s}^{-1}$. Table 1 lists the molecular rate coefficients obtained for the dried, partially rehydrated sample and the aqueous sample in $0.66 \mathrm{M} \mathrm{NaCl}, \mathrm{pH} 8.2,22{ }^{\circ} \mathrm{C}$ (Khoroshyy et al. 2013). Comparison of the rate coefficients shows that while transitions up to the $\mathrm{pB}_{2}$ intermediate are faster in the aqueous sample, those after the $\mathrm{pB}_{2}$ intermediate are up to an order of magnitude slower. 
Table 1 Molecular rate coefficients obtained from the global spectrotemporal fit of the photocycle scheme (Fig. 3) to the present data (rehydrated) and to the $0.66 \mathrm{M} \mathrm{NaCl}, \mathrm{pH} 8.2$ data (Khoroshyy et al. 2013) (aqueous)

\begin{tabular}{lll}
\hline Transition & \multicolumn{2}{l}{ Rate coefficient $\left(\mathrm{s}^{-1}\right)$} \\
\cline { 2 - 3 } & Rehydrated & Aqueous \\
\hline pR1 to pR2 & $4.8 \cdot 10^{4}$ & $3.7 \cdot 10^{5}$ \\
Reverse & $2.7 \cdot 10^{2}$ & $1.6 \cdot 10^{2}$ \\
pR2 to pB1 & $6.7 \cdot 10^{2}$ & $2.2 \cdot 10^{3}$ \\
Reverse & $1.0 \cdot 10^{3}$ & $1.8 \cdot 10^{3}$ \\
pB1 to pB2 & $7.8 \cdot 10^{2}$ & $1.0 \cdot 10^{3}$ \\
Reverse & $1.6 \cdot 10^{2}$ & $2.2 \cdot 10^{2}$ \\
pB2 to pG1 & 16 & 2.9 \\
Reverse & 7.9 & 0.19 \\
pG1 to pG & 2.9 & 0.21 \\
\hline
\end{tabular}

\section{Light-induced refractive index change of the photoactive yellow protein}

With the pulsed excitation, the refractive index change of the sample was determined by monitoring the outcoupled light intensity at an incident angle of the measuring beam (HeNe, $\lambda=632.8 \mathrm{~nm}$ ) tuned slightly off-resonance, to the half-maximum of the peak intensity. Measurements were taken on both sides of the resonance peak, and the absolute values were averaged. The experiments were performed at low- $(20 \%)$ and high- $(75 \%, 85 \%)$ relative humidity environments. For each different environment, the change in refractive index was monitored at two timescales $(50 \mathrm{~ms}$ and $2 \mathrm{~s}$ full scales) after excitation. An additional measurement was taken on the sample at $85 \%$ relative humidity and $500 \mu$ s time scale, for the comparison of the OMA and OWLS results regarding the fast transition in the first part of the photocycle.

At low humidity (20\%, data not shown) and $50 \mathrm{~ms}$ after excitation, the decay of the signal (guided light intensity change after the excitation of the PYP layer) was still in progress, and the calculated relative refractive index change was $\sim 10^{-5}$, which is comparable to the accuracy of our OWLS set-up. By $2 \mathrm{~s}$ after excitation, the measured intensity change was negligible. Comparing the OWLS results to the measured kinetics of the PYP, we concluded that the experienced refractive index change at low humidities stems from the heat jump induced by the exciting light, rather than the protein entering the photocycle. This observation is in agreement with the results of van der Horst et al. (2005).

At high humidities (both at $75 \%$ and $85 \%$, Fig. 5), after $50 \mathrm{~ms}$ from the excitation a quasi-steady state is formed, whose refractive index is different from that of the ground state. The measured kinetics and concentrations at $85 \%$ relative humidity (shown in Fig. 4b) imply that at $50 \mathrm{~ms}$ after
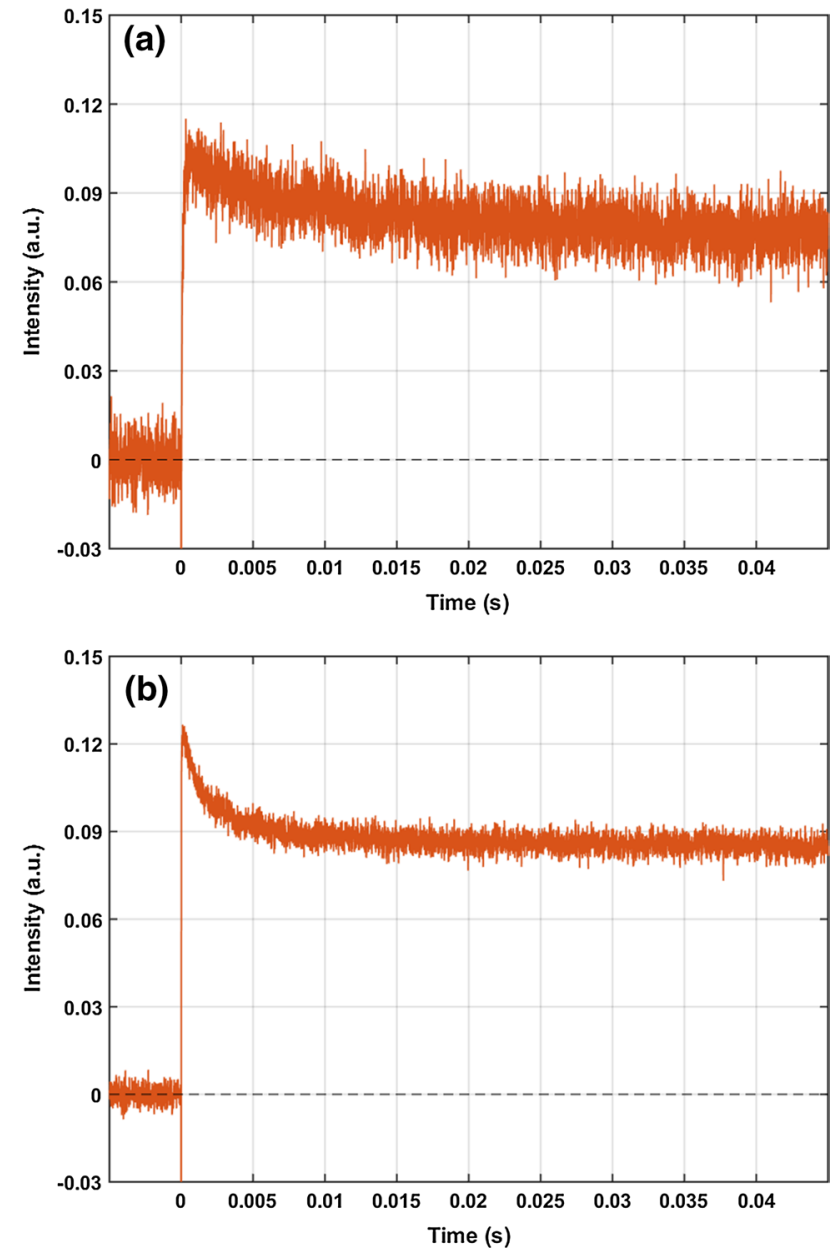

Fig. 5 The measured OWLS data at 75\% RH (a) and $85 \%$ RH (b). The data indicated are measured at the right side of the incoupling peak

the excitation the mixture of two $\mathrm{pB}$ states dominates the photocycle, while the $\mathrm{pR}$ and $\mathrm{pG}_{1}$ conformations are present only in lesser amounts. The calculated refractive index change for the $\mathrm{pB}$ mixture state is $\Delta n=-3 \cdot 10^{-4}$. At $2 \mathrm{~s}$ after excitation, a small residual refractive index signal was present (Fig. 6c), which is likely due to a temperature artefact. At lower, $75 \% \mathrm{RH}$, the results were similar to those obtained at $85 \% \mathrm{RH}$.

At $85 \%$ relative humidity, an additional experiment was done on the 500- $\mu$ s timescale, to compare the kinetic coefficients obtained from the OWLS and OMA experiments. The $500-\mu$ s timescale OWLS data were fitted with two exponentials, the second of them corresponding to the second phenomenological rate constant from the OMA results (Fig. 6a), while the first rate constant was found slightly slower than its absorption kinetic counterpart $\left(2.4 \cdot 10^{4} \mathrm{~s}^{-1}\right)$. For the traces of 50-ms timescale, two exponentials were used, whose kinetic constants corresponded to the second and third phenomenological rate coefficients $\left(7.0 \cdot 10^{2}, 1.44 \cdot 10^{2} \mathrm{~s}^{-1}\right.$, 

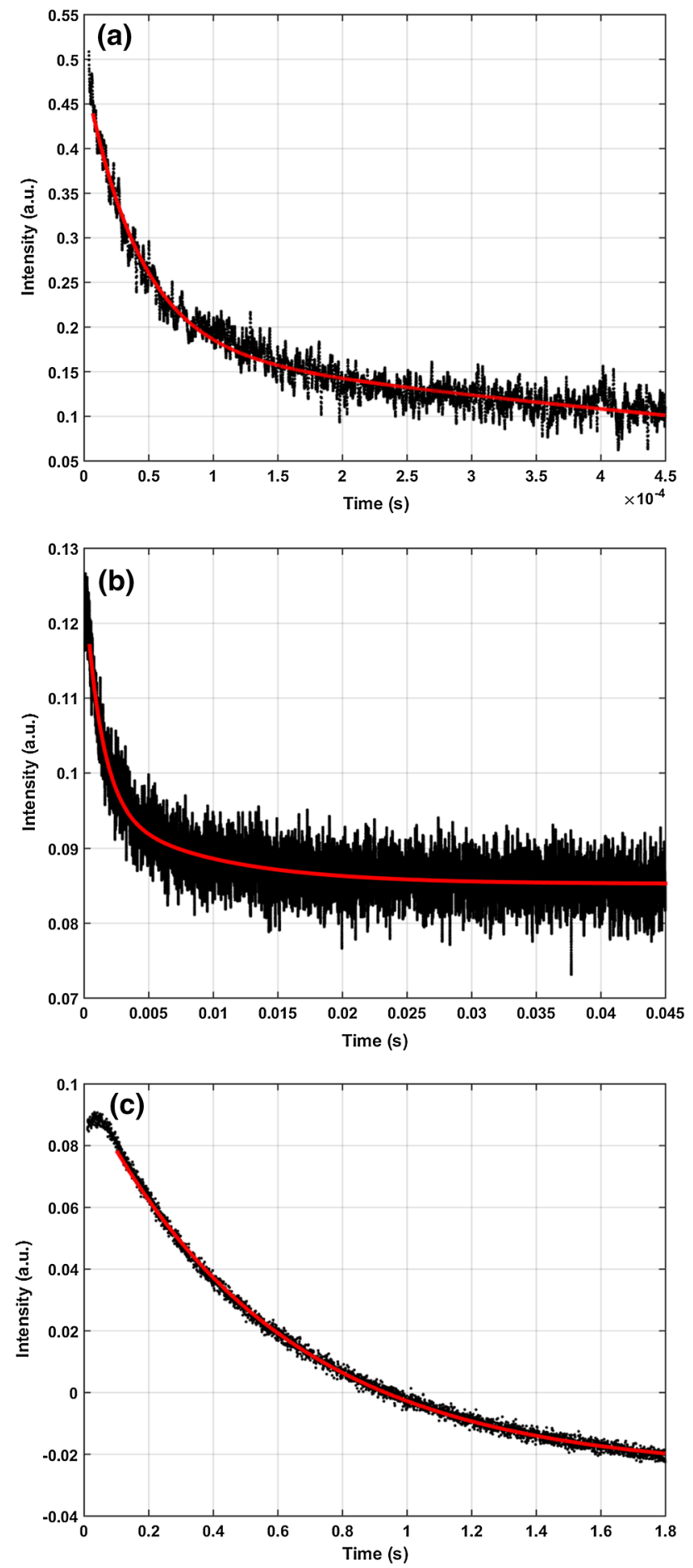

Fig. 6 Exponential fit on the data measured in $85 \%$ RH environment on a 500- $\mu$ s (a), 50-ms (b) and 2-second (c) timescale

respectively, Fig. 6b), while the 2-s timescale data could be fitted with one exponential with a rate constant of $1.7 \mathrm{~s}^{-1}$ (Fig. 6c), which corresponds to the last phenomenological rate coefficient obtained from the OMA's multiexpoential fit.
A global multiexponential fit to the OWLS and OMA data on the 500- $\mu \mathrm{s}, 50-\mathrm{ms}$ and 2-s timescales showed a similar correlation between the kinetics of the curves measured by the two methods, with a goodness of fit of $R^{2} 0.96,0.83$ and 0.99 , respectively, for the three timescales. These results confirm that the refractive index changes of the films are due to conformational changes in the protein molecule following light excitation.

At $85 \%$ relative humidity, an additional experiment with a blue-light $\mathrm{CW}$ excitation was also performed, where a refractive index change $\Delta n_{\mathrm{pB}-\mathrm{pG}}=-5 \cdot 10^{-4}$ was observed at $632.8 \mathrm{~nm}$ (data not shown), and was attributed to a dynamic equilibrium between the $\mathrm{pG}$ and $\mathrm{pB}$ states. This value is slightly higher than the corresponding refractive index change observed during the photocycle $\left(-3 \cdot 10^{-4}\right)$, which might be explained by the $<1$ quantum efficiency of the photocycle for a short flash, similarly to the BR-M transition in bacteriorhodopsin, where stationary excitation of the film yielded higher concentrations of the $\mathrm{M}$ form (Dér et al. 2007). Based on the amplitude ratios of the kinetic OWLS traces (Fig. 6a, b), the transient refractive index changes corresponding to the $\mathrm{pR}_{1}$ and $\mathrm{pR}_{2}$ states are estimated to be even higher (about 10 and 5 times, respectively), which are comparable to the maximum values obtained for bR films.

\section{Conclusions}

The photocycle of dried PYP films was examined at different relative humidities. For determining the kinetics of the photocycle, we used an optical multichannel analyser setup and compared the data to those of light-induced relative refractive index change measurements by optical waveguide lightmode spectroscopy.

At low humidity (20\%), no regular photocycle takes place in the protein, and the small spectral changes observed are probably due to a temperature jump effect caused by the pumping laser.

At high humidities ( $75 \%$ and $85 \% \mathrm{RH}$ ), the photocycle is similar to the one measured in solution, but the rate-limiting steps are faster. In previous publications, a number of different photocycle schemes have been proposed for PYP in solution. According to our analysis, the simple, unbranched photocycle scheme containing multiple red- and blue-shifted intermediates $\left(\mathrm{pR}_{1}, \mathrm{pR}_{2}, \mathrm{pB}_{1}, \mathrm{pB}_{2}\right.$ and $\mathrm{pG}_{1}$ ) with reversible transitions between most of the states, as established by (Khoroshyy et al. 2013), could adequately account for the photocycle of PYP in dried film at high RH (85\%). With the time resolution of the experiments, the formation of $\mathrm{pR}_{2}$ from the $\mathrm{pR}_{1}$ state is the fastest transition with a phenomenological rate constant greater than $4.8 \cdot 10^{4} \mathrm{~s}^{-1}$. The spectra of the two pR states are similar to each other; however, they have sharper maxima than their counterparts in solution. 
The decay of $\mathrm{pR}$ and the formation of $\mathrm{pB}$ states are slower in the dried protein, with the $\mathrm{pB}$ states being less blue-shifted, and $\mathrm{pB}_{1}$ having spectral features resembling a mixture of, presumably, protonated and deprotonated chromophores. The refractive index change of blue-shifted intermediates is calculated to be $\Delta n=-3 \cdot 10^{-4}$, while the faster phases of the OWLS signal indicate refractive index changes in the $10^{-3}$ regime, similar to the highest values of bR-based films.

The above results are expected to serve with essential information for future biophotonic devices utilizing the nonlinear optical properties of PYP or other chromoproteins. Note that optical film preparation at controlled $\mathrm{pH}$ and relative humidity is solved for chromoproteins like bR (see examples in Dér and Keszthelyi 2001 and references therein). According to our experience, optical-quality films of PYP, prepared at pre-adjusted $\mathrm{pH}$, and stored under controlled relative humidity conditions, keep their functionality for at least 6 months. PYP could substitute or complement bR in such applications where, for example, shorter-wavelength operations are needed. Another distinct feature of PYP is that, contrary to bR, it is inherently water soluble in its monomeric form that allows its penetration into nanometric holes of porous silicon or other porous materials, making unique hybrid nonlinear optical structures possible. Most of all, applications in holography or integrated photonics are envisaged.

Acknowledgements Open access funding provided by MTA Biological Research Centre (MTA SZBK). This work was supported by grants from the Hungarian Ministry for National Economy (Economic Development and Innovation Operational Programme, GINOP-2.3.2-15-201600001) and by the National Research, Development and Innovation Office (NKFIH K-124922 and K-108697). The authors are indebted to Dr. John Fitch for generously providing photoactive yellow protein.

Open Access This article is distributed under the terms of the Creative Commons Attribution 4.0 International License (http://creativeco mmons.org/licenses/by/4.0/), which permits unrestricted use, distribution, and reproduction in any medium, provided you give appropriate credit to the original author(s) and the source, provide a link to the Creative Commons license, and indicate if changes were made.

\section{References}

Baca M, Borgstahl GEO, Boissinot M, Burke PM, Williams DR, Slater KA, Getzoff ED (1994) Complete chemical structure of photoactive yellow protein: novel thioester-linked 4-hydroxycinnamyl chromophore and photocycle chemistry. Biochemistry 33:1436914377. https://doi.org/10.1021/bi00252a001

Borucki B, Joshi CP, Otto H, Cusanovich MA, Heyn MP (2006) The transient accumulation of the signaling state of photoactive yellow protein is controlled by the external $\mathrm{pH}$. Biophys J 91:2991-3001. https://doi.org/10.1529/biophysj.106.086645

Cusanovich MA, Meyer TE (2003) Photoactive yellow protein: a prototypic PAS domain sensory protein and development of a common signaling mechanism. Biochemistry 42:4759-4770. https://doi. org/10.1021/bi020690e
Dér A, Keszthelyi L (2001) Bioelectronic applications of photochromic pigments. IOS Press, Amsterdam

Dér A, Valkai S, Fábián L, Ormos P, Ramsden JJ, Wolff EK (2007) Integrated optical switching based on the protein bacteriorhodopsin. Photochem Photobiol 83:393-396. https://doi. org/10.1562/2006-06-21-RA-944

Fábián L, Wolff EK, Oroszi L, Ormos P, Dér A (2010) Fast integrated optical switching by the protein bacteriorhodopsin. Appl Phys Lett 97:023305. https://doi.org/10.1063/1.3462940

Fábián L et al (2011) Protein-based ultrafast photonic switching. Opt Express 19:18861-18870. https://doi.org/10.1364/OE.19.018861

Fábián L, Mathesz A, Dér A (2015) New trends in biophotonics. Acta Biol Szeged 59(Suppl. 2):189-202

Groot ML, van Wilderen LJGW, Larsen DS, van der Horst MA, van Stokkum IHM, Hellingwerf KJ, van Grondelle R (2003) Initial steps of signal generation in photoactive yellow protein revealed with femtosecond mid-infrared spectroscopy. Biochemistry 42:10054-10059. https://doi.org/10.1021/bi034878p

Hales JM et al (2010) Design of polymethine dyes with large thirdorder optical nonlinearities and loss figures of merit. Science 327:1485-1488

Haque SA, Nelson J (2010) Toward organic all-optical switching. Science 327:1466-1467

Hellingwerf KJ, Hendriks J, Gensch T (2003) Photoactive yellow protein, a new type of photoreceptor protein: will this "yellow lab" bring us where we want to go? J Phys Chem A 107:10821094. https://doi.org/10.1021/jp027005y

Hendriks J, van Stokkum IHM, Hellingwerf KJ (2003) Deuterium isotope effects in the photocycle transitions of the photoactive yellow protein. Biophys J 84:1180-1191. https://doi. org/10.1016/S0006-3495(03)74932-7

Hoff WD et al (1994a) Thiol ester-linked p-coumaric acid as a new photoactive prosthetic group in a protein with rhodopsin-like photochemistry. Biochemistry 33:13959-13962. https://doi. org/10.1021/bi00251a001

Hoff WD et al (1994b) Measurement and global analysis of the absorbance changes in the photocycle of the photoactive yellow protein from Ectothiorhodospira halophila. Biophys J 67:1691-1705

Hristova SG, Varo G, Der A (1984) Long time stability of purple membranes from Halobacterium halobium. Acta Biochim Biophys Acad Sci Hung 19:215-219

Hu X, Jiang P, Ding C, Yang H, Gong Q (2008) Picosecond and lowpower all-optical switching based on an organic photonic-bandgap microcavity. Nat Photonics 2:185

Ihee $\mathrm{H}$ et al (2005) Visualizing reaction pathways in photoactive yellow protein from nanoseconds to seconds. Proc Natl Acad Sci USA 102:7145-7150. https://doi.org/10.1073/pnas.0409035102

Imamoto Y, Kataoka M (2007) Structure and photoreaction of photoactive yellow protein, a structural prototype of the PAS domain superfamily. Photochem Photobiol 83:40-49. https://doi. org/10.1562/2006-02-28-ir-827

Joshi CP, Borucki B, Otto H, Meyer TE, Cusanovich MA, Heyn MP (2005) Photoreversal kinetics of the I1 and I2 intermediates in the photocycle of photoactive yellow protein by double flash experiments with variable time delay. Biochemistry 44:656-665. https ://doi.org/10.1021/bi0481141

Khoroshyy P, Dér A, Zimányi L (2013) Effect of Hofmeister cosolutes on the photocycle of photoactive yellow protein at moderately alkaline pH. J Photochem Photobiol B 120:111-119. https://doi. org/10.1016/j.jphotobiol.2012.12.014

Kim TW et al (2012) Protein structural dynamics of photoactive yellow protein in solution revealed by pump-probe X-ray solution scattering. J Am Chem Soc 134:3145-3153. https://doi.org/10.1021/ ja210435n 
Mathesz A et al (2013) High-speed integrated optical logic based on the protein bacteriorhodopsin. Biosens Bioelectron 46:48-52. https://doi.org/10.1016/j.bios.2013.02.022

Meyer TE (1985) Isolation and characterization of soluble cytochromes, ferredoxins and other chromophoric proteins from the halophilic phototrophic bacterium Ectothiorhodospira halophila. Biochem Biophys Acta 806:175-183. https://doi.org/10.1016/00052728(85)90094-5

Meyer TE, Yakali E, Cusanovich MA, Tollin G (1987) Properties of a water-soluble, yellow protein isolated from a halophilic phototrophic bacterium that has photochemical activity analogous to sensory rhodopsin. Biochemistry 26:418-423. https://doi. org/10.1021/bi00376a012

Meyer TE, Tollin G, Hazzard JH, Cusanovich MA (1989) Photoactive yellow protein from the purple phototrophic bacterium, Ectothiorhodospira halophila. Quantum yield of photobleaching and effects of temperature, alcohols, glycerol, and sucrose on kinetics of photobleaching and recovery. Biophys J 56:559-564. https:// doi.org/10.1016/S0006-3495(89)82703-1

Mix LT et al (2018) Excitation-wavelength-dependent photocycle initiation dynamics resolve heterogeneity in the photoactive yellow protein from Halorhodospira halophila. Biochemistry 57:17331747. https://doi.org/10.1021/acs.biochem.7b01114

Moore GE (1965) Cramming more components onto integrated circuits. Electronics 38:114-117

Oesterhelt D, Stoeckenius W (1971) Rhodopsin-like protein from the purple membrane of Halobacterium halobium. Nat New Biol 233:149

Oesterhelt D, Stoeckenius W (1973) Functions of a new photoreceptor membrane. Proc Natl Acad Sci 70:2853-2857

Ormos P, Fábián L, Oroszi L, Wolff EK, Ramsden JJ, Dér A (2002) Protein-based integrated optical switching and modulation. Appl Phys Lett 80:4060-4062. https://doi.org/10.1063/1.1481197

Pande K et al (2016) Femtosecond structural dynamics drives the trans/ cis isomerization in photoactive yellow protein. Science 352:725729. https://doi.org/10.1126/science.aad5081

Service RF (1995) Two steps for light-altering polymers. Science (New York, NY) 268:1570
Sprenger WW, Hoff WD, Armitage JP, Hellingwerf KJ (1993) The eubacterium Ectothiorhodospira halophila is negatively phototactic, with a wavelength dependence that fits the absorption spectrum of the photoactive yellow protein. J Bacteriol 175:30963104. https://doi.org/10.1128/jb.175.10.3096-3104.1993

Ujj L, Devanathan S, Meyer TE, Cusanovich MA, Tollin G, Atkinson GH (1998) New photocycle intermediates in the photoactive yellow protein from Ectothiorhodospira halophila: picosecond transient absorption spectroscopy. Biophys J 75:406-412. https ://doi.org/10.1016/S0006-3495(98)77525-3

Van Beeumen JJ et al (1993) Primary structure of a photoactive yellow protein from the phototrophic bacterium Ectothiorhodospira halophila, with evidence for the mass and the binding site of the chromophore. Protein Sci 2:1114-1125. https://doi.org/10.1002/ pro. 5560020706

van der Horst MA, van Stokkum IHM, Dencher NA, Hellingwerf KJ (2005) Controlled reduction of the humidity induces a shortcut recovery reaction in the photocycle of photoactive yellow protein. Biochemistry 44:9160-9167. https://doi.org/10.1021/bi050237d

Waldrop MM (2016) The chips are down for Moore's law. Nat News 530:145-147

Yang C, Kim SO, Kim Y, Yun SR, Choi J, Ihee H (2017) Photocycle of photoactive yellow protein in cell-mimetic environments: molecular volume changes and kinetics. J Phys Chem B 121:769-779. https://doi.org/10.1021/acs.jpcb.6b13076

Yeremenko S, van Stokkum IHM, Moffat K, Hellingwerf KJ (2006) Influence of the crystalline state on photoinduced dynamics of photoactive yellow protein studied by ultraviolet-visible transient absorption spectroscopy. Biophys J 90:4224-4235. https://doi. org/10.1529/biophysj.105.074765

Publisher's Note Springer Nature remains neutral with regard to jurisdictional claims in published maps and institutional affiliations. 